\title{
Contribution of Virtual Reality for Lines Production's Simulation in a Lean Manufacturing Environment
}

\author{
Mohamed-Amine Abidi, Barbara Lyonnet, Pierre Chevaillier, and Rosario Toscano
}

\begin{abstract}
In an increasingly competitive world, the responsiveness has become essential to improve the performance of companies. Accordingly a large number of companies have implemented the Lean approach. This approach is a production management method based on waste elimination and just-in-time production. This leads manufacturers to update and adapt their ways of production management and so reorganize the production lines to reduce costs, minimize delays as well as optimize their flexibility. To do this, the use of the Virtual Reality (VR) in the lines production's simulation would offer many advantages. Thus, this technology would allow users to interact with simulation model in real-time and in a 3D format. However, few works have integrated virtual reality in the field of simulation in Lean manufacturing environment. The objective of this paper is to propose a method of simulation of production flows in a virtual environment in real time. To this end, we will review the traditional industrial tools in the field of production simulation. Then we will present the main advantages of using the VR to the manufacturing processes in a lean environment. Finally a global software solution which integrates VR will be proposed and an example of its application to simulate lines production in VR will be presented.
\end{abstract}

Index Terms-Virtual manufacturing, production line simulation, virtual reality, 3D simulation.

\section{INTRODUCTION}

To remain competitive companies must continuously find innovative solutions to improve various industrial processes such as manufacturing and monitoring processes. In this context, the Lean approach has emerged as an ideal solution.

The term "Lean production" was used for the first time in 1988 by Krafcik [1] to describe the Toyota Production System (TPS). The Lean approach is a production management method to improve performance based on waste elimination and just-in-time production. By the late 1980s the Lean approach has been applied in the worldwide automotive industry and its subcontractors [2]. The spectacular results of this approach in terms of increased productivity, reducing costs and time have aroused great interest in businesses [3]. Many industries, including aerospace, electronics and mass consumption implemented the Lean production [4]. Lean

Manuscript received November 30, 2014; revised February 3, 2015.

Mohamed-Amine Abidi and Rosario Toscano are with National Engineering School of Saint-Etienne, France (e-mail: mohamed-amine.abidi@enise.fr, rosario.toscano@enise.fr).

Barbara Lyonnet is with Nantes University, France (e-mail: barbara.lyonnet@univ-nantes.fr).

Pierre Chevaillier is with National Engineering School of Brest, France (e-mail: pierre.chevaillier@enib.fr). approach is intensively implemented, in both small [5] and large companies [6]. Thus, today the Lean approach seems to be an ideal solution for a vast majority of companies.

Lean system requires the development of flexible resources to respond quickly to changes in demand [7]. Thus, Lean approach could enable companies to growth in fully-expanding markets with unexpected changes. According to [8], a production system Lean-Agile is based on the use of new technologies, information Systems (TIC, EDI), enhancing innovation capacity throughout production chain and forms of virtual enterprises. The information systems are emerging as a cornerstone to improving the performance and responsiveness.

Thus, since the 90s, the increasing diversity and complexity of products has gradually led manufacturers to use computer software in various stages of production, to enable manufacturers to control management systems and also to simulate various processes of production systems. In recent decades, the IT (Information technology) world saw a technological revolution. Nowadays, many softwares were developed throughout the life-cycle of the product. To use these softwares, it is necessary to have theoretical and technical expertise in industrial engineering. However, the companies do not always have these skills.

To facilitate the use of developed softwares in industry, a new generation of industrial simulation's systems appears, wherein the integration of virtual reality (VR) and 3D technology is essential. In this area, there are many academic and industrial laboratories that develop their own techniques and tools for research projects for industry. These new tools for integrating VR techniques are used to provide a realistic view of a manufacturing process, but also to visualize the normal operation of a manufacturing process to $3 \mathrm{D}$. In addition, VR techniques allow to the user to configure an industrial process via a motor-sensory interface and visualize the progress of the process. Several researchers are working on this issue to revolutionize the field of industrial process simulation [9]-[11].

In the context of improving responsiveness, simulation tools associated to those developed by the VR offer promising perspectives to improve production systems. The optimization of material flow is a current key issue of the organization of production systems. One of the essential principles of Lean is based on the optimization of production flows by the principle of just in time and elimination of wastes. A tool to visualize flows, to detect stocks and queues from production simulations, should lead to a better design of the system and to anticipate the wrong technical and technological choices. As recommended by Lean production, flexibility and responsiveness of flows are fundamental bases. 
The VR technology allow to take action upstream, at the very start of design and so have production systems more adapted to the various constraints of enterprises.

Currently to our knowledge, there are few works on the contribution of VR for simulations of production lines in a lean manufacturing environment. Yet the integration of VR techniques could provide considerable improvement of visualization and optimization of manufacturing flow.

This paper shows the interest of integrating VR techniques for simulating production lines. A literature review of the main tools of simulating production lines will be presented. An analysis of major research projects that integrate the VR in new generation of simulation systems will be proposed. Finally, we propose the first model coupling between an ARENA simulation model and a virtual environment in real time. This model could help to address the challenges of responsiveness and flexibility that companies currently encounter in Lean environment.

\section{Simulation of Production Lines AND Literature REVIEW}

\section{A. Existing Tools of Lines Production's Simulation}

There are several types of traditional industrial tools share the same common goal, which is the automation of manufacturing facilities and computerization of various functions of production, such as: simulation, modeling, workflow management of production, design and logistics. The simulation of a production system is the fact of playing with the various system parameters such as the size of the storage areas, priority rule etc., to arrive at a better configuration. In this section we present some traditional tools specialized in simulation of production flow. We will analyze the fundamental of functioning used in these tools. Among the most used tools in modeling and simulation of manufacturing processes, we find that ARENA tool did not cease to evolve since the late $1990 \mathrm{~s}$

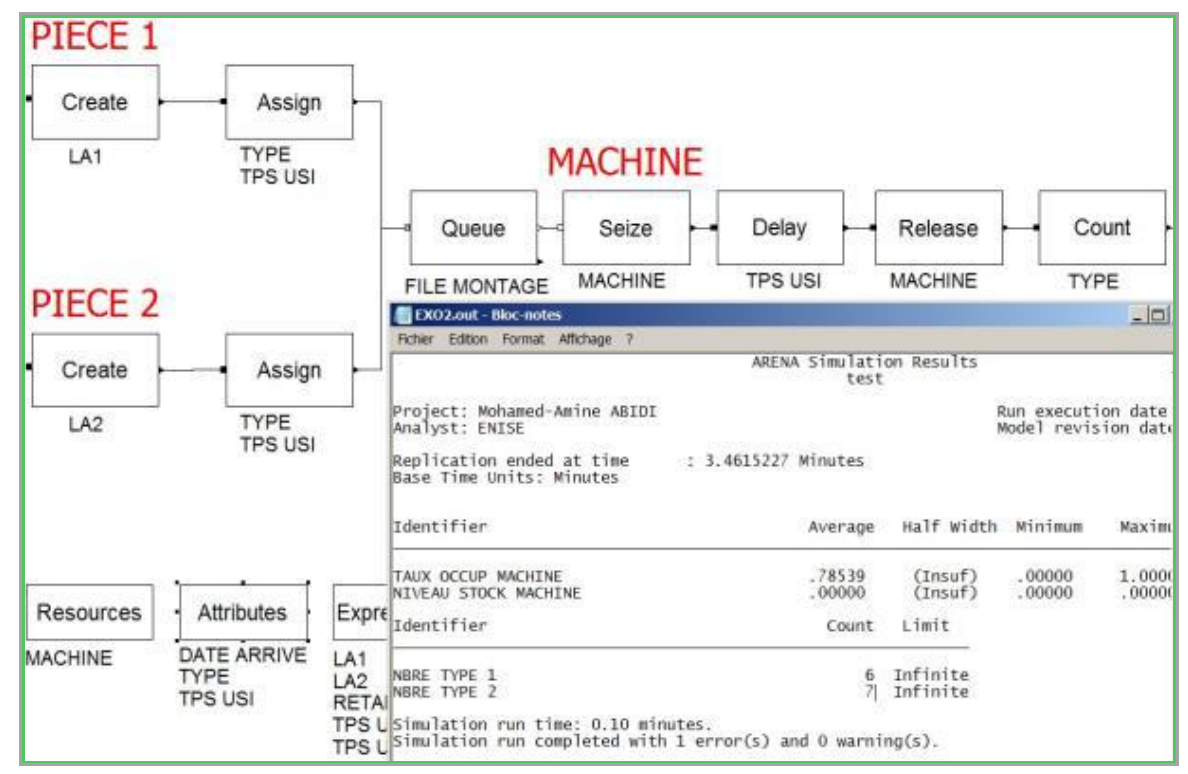

Fig. 1. Example of industrial model with ARENA [12].

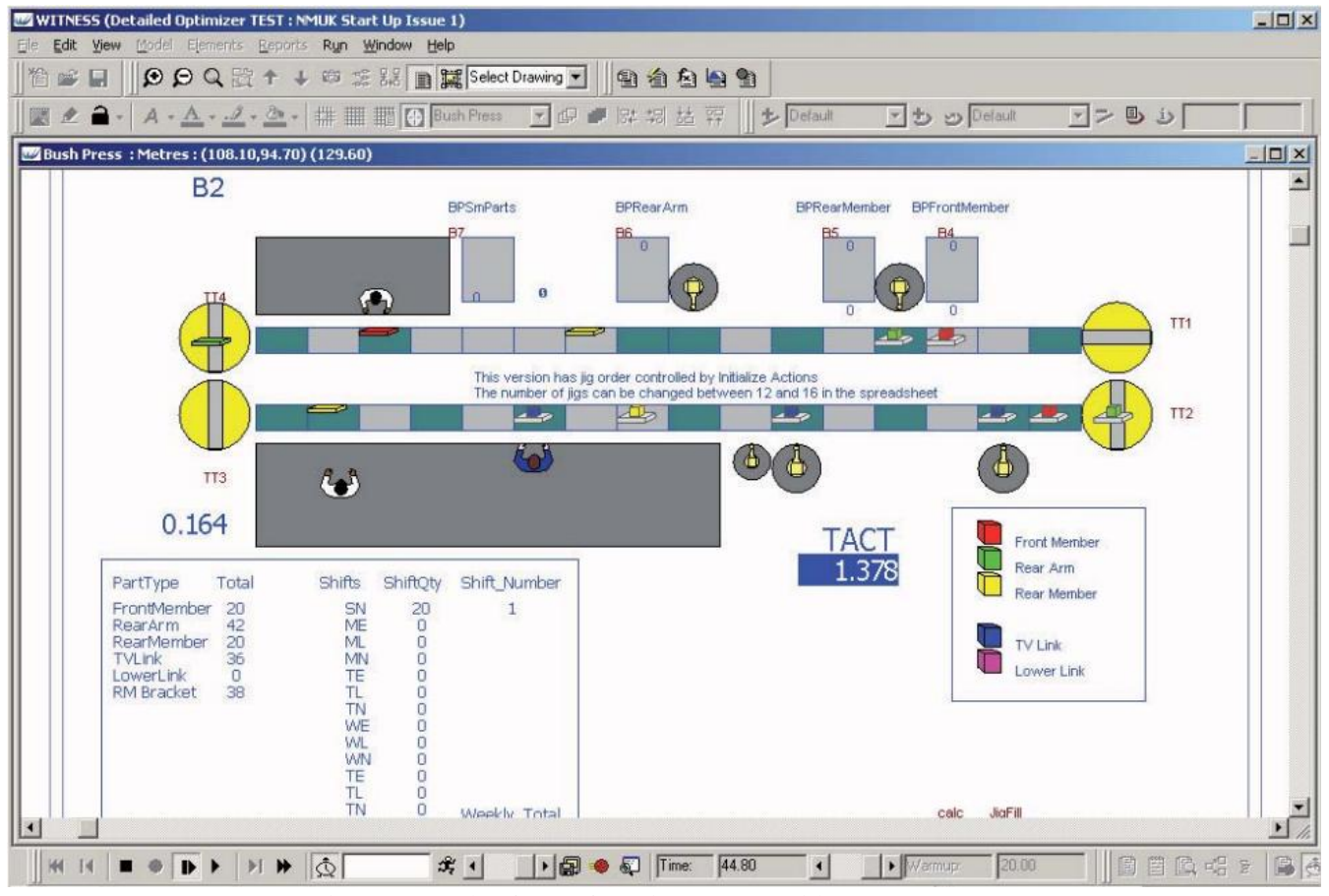

Fig. 2. Example of manufacturing process with WITNESS [14]. 
ARENA allows to the industrial engineers to create a simulation model that reflects the operation of a concrete industrial process. ARENA [12] is based on object-oriented design to build a graphical model. An ARENA model consists of different blocks that represent the real components of an industrial system. As per the authors of Dias et al. [13], The ARENA Simulation is one of the most popular and powerful simulation software in modelling an automated manufacturing process.

There are many other simulation software of manufacturing flows. Among them we can mention WITNESS. An example of manufacturing process with WITNESS is presented (see Fig. 2). This software allows users to model and simulate the manufacturing processes. Following the flexibility and openness of simulation tools available today, we can now control all aspects of the organization of industrial engineering and manufacturing [14]. WITNESS is composed of several modules such as: Matflow (a system of flow planning) WITNESS Optimizer.

Today, the latest versions of the industrial simulation softwares begin to integrate 3D technology. For example, the QUEST tool [15] includes a viewer that can present an industrial system in 3D. QUEST is always proposed with DELMIA. The combination of tools QUEST/DELMIA [15] is used to help the aerospace, automotive and other areas such as teaching in universities and to strengthen the culture of competitiveness in the aerospace industry. QUEST offers a unique collaborative environment for industrial and manufacturing engineers, QUEST provides the best management practices workflow throughout the industrial process. These tools have been applied in most cases to represent flows in 2D or 3D format, this representation is an animated static film. However, these tools do not allow the user to interact with a simulation model in real-time.

\section{B. Simulation and Lean Manufacturing}

Many works in simulation were reported in the field of Lean Manufacturing [16]. For example, McDonald Described an application of an important tool in the Lean approach, the Value Stream Mapping and simulation to a dedicated product line in an engineer-to-order motion control products manufacturing plant. They used ARENA for the purpose of simulation [17]. [18] Developed a simulation in ARENA to model a factory in China that produces oval-gear flow meters to understand the effect of implementing lean control approaches in the factory. Abdulmalek et al. [19] described a simulation model that was developed to contrast the "before" and "after" scenarios of VSM constructed for a large integrated steel mill.

Although significant works have been carried out in the recent past in the areas of simulation in Lean manufacturing environment, most studies have not integrated virtual reality. Yet, the use of Virtual Reality has many advantages. One of them is that the Virtual Reality allows representing the industrial process in 3D format. Also, this technology allows users to interact with simulation model in real-time. We introduce the virtual reality technology and its contributions to the simulation of industrial processes in the following section.

\section{Virtual Reality and Its Contribution to the Simulation of Industrial Processes}

In this section, we introduce the virtual reality technology and its contributions to the simulation of industrial processes through examples of previous research works that address issues related to the industry.

The Virtual reality as defined by Arnaldi [20], is "Scientific and technical domain exploiting computer science and behavioral interfaces to simulate in a virtual world the behavior of $3 D$ entities that interact in real time with each other and with one or more users in pseudo-natural immersion by means of sensorimotor channels". The immersion and interaction present the most important phenomena for applications VR, for this we should implement techniques which allow to immerse the user in a virtual environment via sensory-motor interfaces whose purpose is to deal with virtual processes. The user receives a set of information through these sensory-motor interfaces such as: return immersive 3D on a big screen, force feedback through a haptic arm which reflecting the collision with virtual objects etc. The interaction with the virtual scene requires an interface of sensorimotor control as haptic arms for seizure of virtual objects, the motion capture system which can control a virtual avatar, data gloves etc. Often considered as an extension of the simulation, the VR is distinguished by the fact that it offers a good level of immersion (display, touch etc.) to users. The VR allows the modern industry to simulate industrial processes in an efficient manner; it gives the opportunity to interact with the simulation model. A virtual simulation system includes a set of sensory-motor interfaces and controls the behavior of simulation in comparison with models programmed.



Fig. 3. Visualization in VR of manufacturing system's simulation [9].

The use of existing tools today for the simulation of various industrial processes requires a good knowledge and expertise in the relevant field. Many industrial actors do not have this purely technical expertise. Therefore to allow them to participate in the analysis, optimization and validation of simulation models of different processes, many researchers sought to develop innovative solutions that allow for an abstraction between the simulation techniques and non-specialist actors. Dangelmaier et al. [9] (see Fig. 3) propose a VR system to visualize a $3 \mathrm{D}$ simulation of 
manufacturing lines. The objectives of this system are to assist users in the design step of production system, validate these models and help optimize a production system.

There are other works that deal with specific issues such as production simulation in aerospace. Caggiano et al. [11] propose an innovative solution for digital simulation. The main feature of this solution is to aid the design and simulation of an existing cell of manufacturing components of aircraft engines to be improved by automated robotic deburring. Why the researchers put in place an application of $3 \mathrm{D}$ motion simulation is to illustrate the design of handling systems of materials. This system uses a discrete event simulation to analyze the different scenarios and improve the performance of the manufacturing cell.

Multiple approaches and specific methods of design engineering and simulation of production processes are possible today thanks to various technologies. Tolio et al. [21] seek to develop a new concept that offers the possibility of integrating various tools for design, simulation and analysis in a single solution. This concept allows supporting interoperability between these tools throughout the life cycle of production. The researchers in the field of architecture are generally concerned with solutions of simulation and learning of construction modes. Goulding et al. [22] propose a VR solution for the simulation and learning of the most important techniques in construction of buildings. The main goal of this solution is to provide a work environment that allows different actors of industry (e.g. project managers, construction managers, architects, designers, suppliers and manufacturers) to have a multidisciplinary learning via a VR simulation of a construction site.

Lee et al. [23] work on a simulation system based on mixed reality to simulate industrial operations in a virtual factory. This system enables manufacturers to launch virtual operations in order to analyze the feasibility of a solution in a real context and see their evolution over time. Leu et al. [24] present in their paper a state of the art which describes the various methods used to develop CAD models and the systems of simulation for the assembly of prototypes, planning and training. They introduced a method for exchanging data between a CAD system and a VR system.

In a recent study addressing the treatment of simulation and control of different stages of PLM, Fillatreau et al. [10] propose a VR solution that allows manufacturers to navigate in a $3 \mathrm{D}$ scene, and manipulate virtual components, whose purpose is to simulate and validate the various operations of all PLM stages. The main objective of this system is to improve the traceability of project reviews, decisions and documentation in all phases of PLM. Mujber et al. [25] propose in their article a classification of industrial applications VR, according to which there are three groups: Design (modeling and prototyping); Operations management (planning, simulation and training) and Manufacturing processes (machining, assembly and inspection). This demonstrates a wide range of demand of VR in the industry. Today the VR is far from reaching all stages of PLM (e.g. validation of product requirements, validation of simulation models, simulation of flow, acceptance of products: are not really addressed by VR applications). The existing studies in the literature that address the integration of VR in different stages of PLM always focus on a particular stage.

Today, in the current market there are tools of simulation and planning of industrial platforms that are already marketed and use 3D technology. These systems are often used for the simulation of the fabrication and the implementation of a plant in an industrial platform. There may be mentioned three commercial products, Tecnomatix Factory FLS by Siemens, Teamcenter Manufacturing Plant Simulation by UGS and MPDS4 Factory Layout by CAD Shroer. These products have a good market share in industry. All this systems are quite similar because they all provide a visual platform with a $3 \mathrm{D}$ rendering. These tools are designed to meet the following industrial needs: design of prototypes, validation of maintenance process and machining, the simulation of the manufacturing process, the evaluation of the establishment of industrial components via virtual platform, the simulation of machining and assembly operations etc. These systems 3D simulation are used to visualize and refine the theoretical results before setting up the real process.

Other researchers are beginning to implement the results of their research in the design and evaluation of manufacturing systems through augmented reality applications.

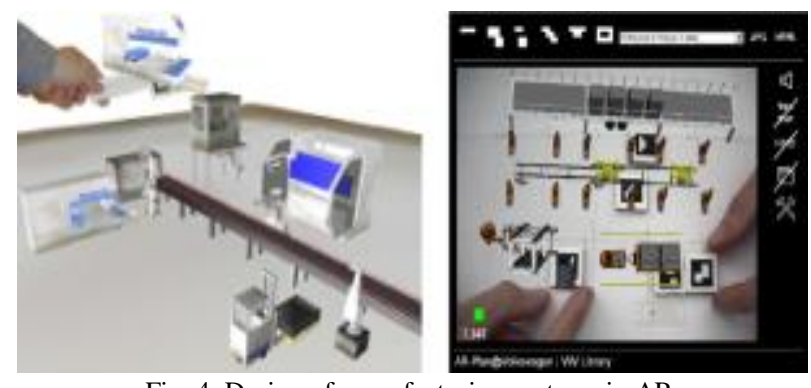

Fig. 4. Design of manufacturing systems in AR.

Gausemier et al. [26] have established a system of AR which allows designers to define and evaluate their models of manufacturing systems utilizing a webcam and markers in a real environment (see Fig. 4. left). Another similar system is presented by Doil et al. [27] (see Fig. 4. right). These systems follow the same principle of VR systems.

\section{Interoperability between Simulation Modules and Semantic Modeling of Virtual Environment (VE)}

With the development of new technologies, several researchers working on the development of technical interoperability between different industrial tools (e.g. modeling, simulation and data representation) based on HLA "High Level Architecture" [28]. The simulations based on HLA interact with each other via a software interface layer, called RTI "Runtime Infrastructure". On the other hand, there are techniques which are based on XML and WS "Web Services" and provide a general interoperability framework on the Web, whose systems (e.g. simulation software) interact through a WS layer, using the mechanism RPC "Remote Procedure Call". The WS layer transports the RPCs in the form of XML messages.

With the arrival of VR technology, the industrial VE can be connected with the rest of the information manufacturing systems that already contains several systems to interoperate. So a software architecture is necessary for combine these heterogeneous systems. 
Other researchers are working on developing simulation frameworks for intelligent VEs. The SCIVE framework [29] provides bidirectional semantic reflection to develop module specific code as well as module independent components. Its inter-module data exchange and synchronization can conveniently be configured on a high level, which includes the definition of general application logic down to per-attribute changes via the filter and rules concept. It provides an interoperability layer allowing semantic data exchange between different heterogeneous sub-systems. There is another framework called VR-WISE [30] "Virtual Reality - With Intuitive Specification Enabled": is a conceptual model-based approach for VR application development. As conceptual modeling will introduce a mechanism to abstract from implementation details, it will reduce the complexity of developing a VE and it avoids that people need a lot of specific VR knowledge for such a conceptual design phase. This framework is specialized in conceptual modeling; it is useful for modeling a mechanical system. But it does not promote interoperability with other systems like the simulators of industrial processes. The MASCARET framework [31] presents a logical connection between the world of system engineering and virtual reality. This framework introduces a layer of abstraction between the $\mathrm{VE}$ and the domain model. It allows to build a semantic representation of the industrial system using the modeling language the expert of this field use, SysML. MASCARET is also an execution platform implemented on various 3D engines, like Ogre or UNITY3D. MASCARET refines and extends SysML with concepts specific to 3D interactive simulation and the modeling of human activities. Behavioral models are based on statecharts and activity-charts. Thus, it allows to simulate the automated or manual operations of an industrial process in the VE.

\section{Methodology And Results}

In this paper, our goal is to simulate the production flow in a VE in real time. Compared to our goal, VR-WISE has a limited interoperability. SCIVE provides an interoperability layer allowing an exchange between the individual modules. The production flow simulation has a specific synchronization mode. This mode allows us to simulate a year of production in less than an hour. Compared to our goal, SCIVE we do not achieve this VE simulation. In this work, our methodology is based on the use of an engine of simulation called SIMAN with ARENA tool. For the exchange of data between ARENA and other heterogeneous modules, we must develop a specific interface. We use the MASCARET framework [31] for the semantic modeling of the $\mathrm{VE}$ and simulation of various entities of the environment.

\section{A. Architecture}

In this part, we present the global architectural design of this proposed system which allows to decompose this system on modules order to better understand its functioning (see Fig. 5). In our approach, the integration of simulation model in a VE requires the creation of a SysML model that reflects the operation of the industrial system based on UML modeling (activity, state and class diagrams). Each of these diagrams presents a specific mechanism, such as the activity diagram showing the flow of activities in a process in different states of the system. After the semantic model validation, we export the XMI file that describes the SysML diagrams. This step is to analyze the diagrams that describe the operation of the model and represent their behavior in VE via MASCARET. After the XMI export, we prepare a description of the 3D scene as XML file.

The principle of operating steps of this system can be summarized in three main steps: (1) make a transformation of the simulation model to a SysML model and export the XMI description. (2) Prepare the MASCARET to analyze the XMI description and map it with the VE. (3) Launch the simulation tool and connect this with the VE through the real time communication infrastructure.

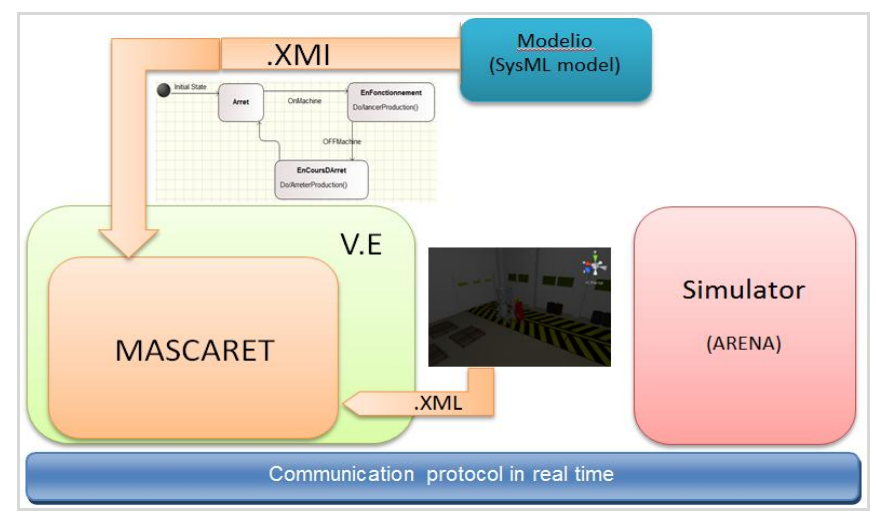

Fig. 5. Architecture of our proposed system.

After transforming the ARENA model in SysML diagrams, this model is imported into MASCARET as an XMI file to reproduce the behaviors of the simulation model in the VE. To synchronize the operation process of both simulation worlds, we created a protocol for data exchange via RTI which allows us to transfer the various simulation data in both directions. When creating entities in the ARENA model, our system publishes all information of each entity in MASCARET via communication protocol (RTI). This allows to start creating $3 \mathrm{D}$ objects corresponding to entities of the simulation model in ARENA, also to apply the behaviors of the simulation on these $3 \mathrm{D}$ objects to get a $3 \mathrm{D}$ simulation. Also, we can communicate the different data of 3D objects in VE with the equivalent entities in simulator. Therefore, we exchanged the simulation data in both directions.

\section{B. Development of Solution}

We coupled the interactive VR system with ARENA simulator, which allows to visualize a simulation of production flows in the course of execution and to interact with it by changing its configuration and its parameters in the VR environment. In this section, we present our method for coupling the different parts of this system (see Fig. 6).

To connect these heterogeneous environments, we used the open source library "OpenSplice" that allows to exchange data between systems of different nature; [OpenSplice: is an open source framework of RTI "Real Time Infrastructure" which facilitates the exchange of data using sockets and programming routines, it is also able to exchange between different machines on a wide area network]. This technology enables the ARENA simulator to communicate with the VE in 
order to have a virtual representation of the simulation model. Also, we can exchange the data in the opposite direction (that is to say from the VE to ARENA), which allows to interact with the simulation model by interacting on the parameters of this model in VE.

To establish a fluid connection between ARENA and RTI (see Fig. 6), we have developed a program that allows to retrieve the different data of model from ARENA and publishing them through the RTI. This program presents an interface between the simulation model and the RTI; we used $\mathrm{C} / \mathrm{C}++$ to develop this interface. The operating principle of the RTI is: it allows different parts of system to subscribe and publish in a data bus. So "ArenaInterface.dll" allows to publish and to subscribe on this data bus. To retrieve data from the simulation model, we have developed routines in "ArenaInterface.dll". These routines allow communicating with the EVENT blocks of simulation model. Each entity going through this block triggers an event. Following this event, the routines are calls for action that retrieves data from different entities.

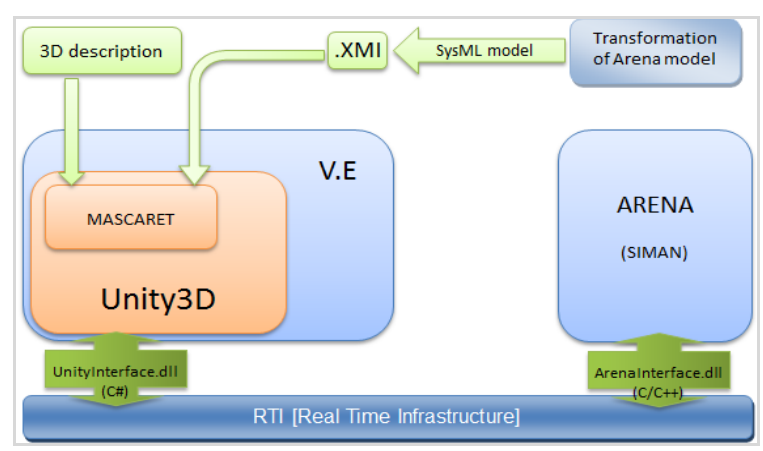

Fig. 6. Technical components of our system.

Similarly, we have developed an interface "UnityInterface.dll" to connect the VE to RTI. We use C\# to develop this interface. To exchange data with the simulation model, we have developed some routines in "UnityInterface.dll". These routines allow subscribing on the data bus; it also allows to retrieve the simulation data in real time using RTI protocol. Our VE contains industrial scenarios that are based on state machines. These machines are operated by MASCARET; they intercept data from triggered events in the simulation model by the EVENT block.

This coupling architecture presents the different parts of our system. To implement this solution, we decomposed this work in three principal steps:

1) Transformation of an ARENA model in SysML model: This step allows to analyze the ARENA model for the purpose of designing a SysML model (SysML model allows to represent the systems in the form class, activity and status diagrams).

2) Development of behavioral simulation in a VE using the MASCARET framework: In this step, it sought to simulate the operation of an industrial process via diagrams class, activity and state machines developed in the first step.

3) Exchange the simulation data between the two worlds: VE and ARENA model via the RTI protocol.

\section{Example of Coupling of VE with ARENA Model}

In this section, we will present a concrete example of coupling an ARENA model with the corresponding VE. On the one hand, Fig. 7 shows the simulation model adapted to be coupled with the $3 \mathrm{D}$ scene according to our architecture.

In Fig. 7, we note the presence of 4 blocks "Event" listed from 1 to 4 . These blocks are used to exchange data between the ARENA model and other components of the architecture via "ArenaInterface.dll". The "Event" number 1 and 2 allowing to communicated to VE settings entities created by blocks "Create". The Event number 3 sends the information of entities (e.g. machining time, type) that go through the machine. The Event number 4 gives the state and some parameter of machined parts.

On the other hand, we transform the ARENA model to a SysML model using a program (.dll) that lets you export it from ARENA as an XMI file. We developed this program (.dll) with VB language. In Fig. 8, we see the UML model that will allow MASCARET to control and represent the behavior of the production system in VE. In the same figure shows the semantic description of the VE in XML.

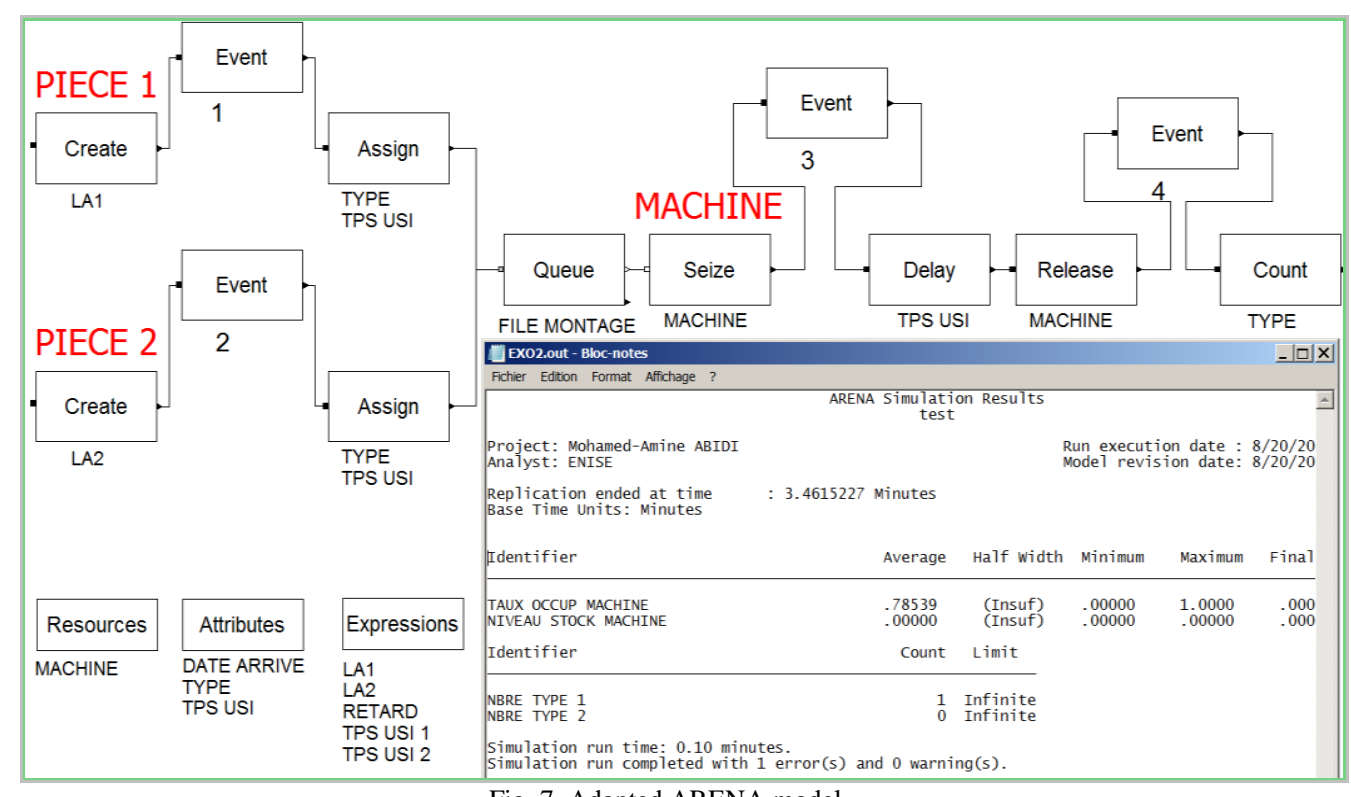

Fig. 7. Adapted ARENA model. 


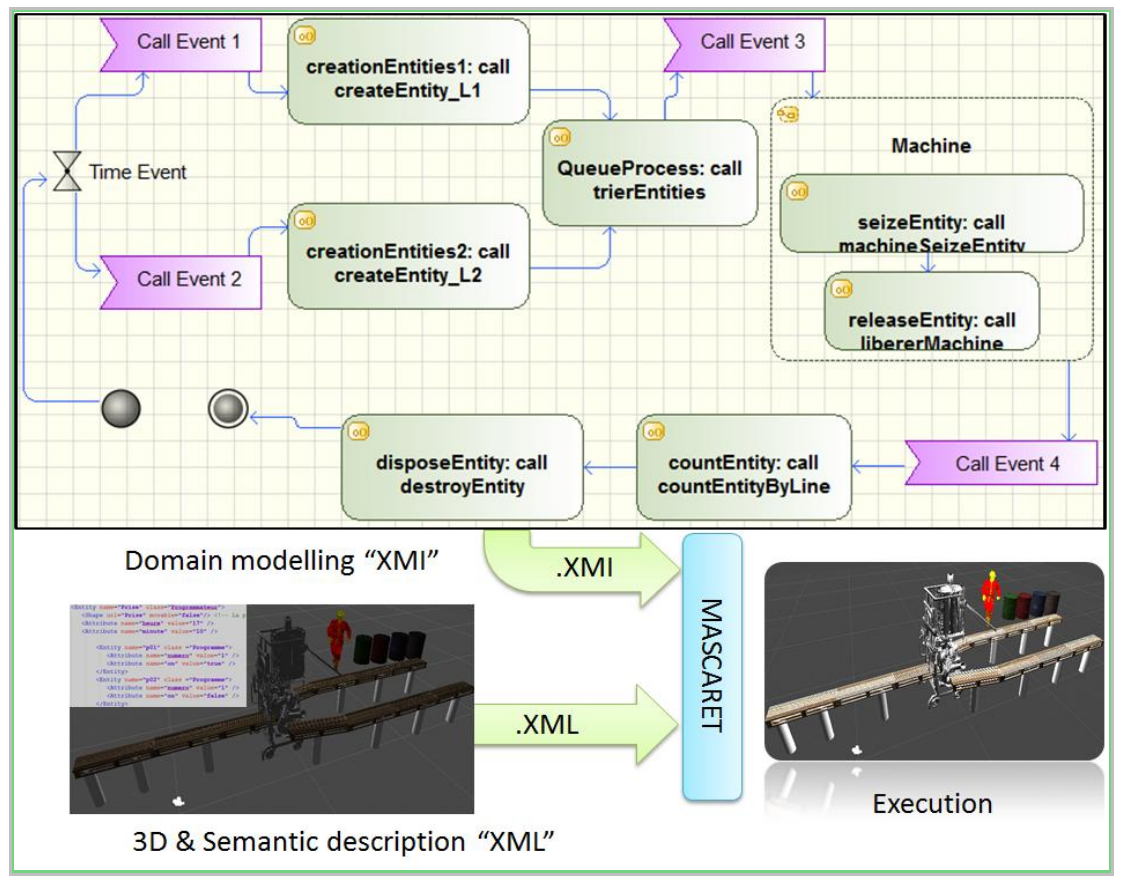

Fig. 8. Coupling of VE with ARENA model.

The communication module in real time based on RTI technology ensures data transfer between the two worlds of simulation. This module allows MASCARET to recover events triggered in ARENA. This connection allows MASCARET to move from one state to another and launch the behaviors that correspond to these states.

\section{DISCUSSION AND CONCLUSION}

Today many companies implement the Lean system to improve their performance. The success of this implementation requires the flexibility of the company, including that one of the lines production. The reorganization of the production lines aims the reduction of costs, the minimization of delays as well as the optimization of their flexibility. To do this, companies began by modeling their production processes using ARENA, they used the same tool to simulate the functioning of their model. This method of work confronts us with our principal problem: it is hard for the various stakeholders to understand or handle complex data resulting from the flow simulation (as mentioned in the first section). Following these needs, we have implemented this solution, which will enable to industrials to have a VR simulation.

Nowadays, virtual reality is used in many industrial settings such as training, decision support, helping with maintenance, simulation of industrial operations, etc. In this paper, a new approach is developed for popularizing the manipulation of simulation of industrial processes. This approach allows to visualize and interact with an industrial simulation. It also allows for the industrial actors to visualize the functioning of their factories. This approach is based on the VR technology and the behavioral programming; this has enabled us to develop an immersive and interactive VR system to bring the user into a 4th dimensions "Sensation" via specific peripheral devices of VR. The main goal of this work is to enable different industry actors to participate in the analysis and improvement of industrial processes. Thus, a French company named "Renault Trucks" is already interested in the results of this work.

In recent years, several research works have been done to virtualize certain industrial processes such as simulation of assembly of pieces, simulation of production flow etc. To deal with such problems, we encountered some technical and scientific obstacles such as: the development of a data exchange module between a simulation tool and VR system, the choice of a part of the simulation to virtualize, the behavioral programming operation of a virtual factory and the transformation of discrete events models to the SysML models.

In addition, the use of this new global software solution integrating VR could be very helpful when companies use the value stream mapping (VSM) to identify the waste reduction opportunities and improvement areas. The first step of VSM is to map out the current state of product and information flow, from raw material to customer for manufacturing processes. After examining this current state and identifying the opportunities in the reduction of lead times, operating costs and the improvement of on-time delivery, the second step is to create a future state and to propose an implementation plan. Our global software solution integrating VR would allow companies to visualize in 3D format the future state and to test and validate their implementation plan. This could facilitate decision-making by companies.

In the future, we aim to develop a VR system for online simulation. This system will connect a VE with an industrial information system (e.g. ERP) and a central system of control/command of machines. The objective of this system is to provide in real-time the current operation of the factory, to analyze and simulate this over time and to take control over the machines of different production lines to reconfigure and improve production processes.

\section{REFERENCES}

[1] J. F. Krafcik, "Triumph of the lean production system," Sloan Management Review, vol. 30, no. 1, pp. 41-52, 1988. 
[2] A. Kochan, "The automotive industry looks for lean production," Assembly Automation, vol. 18, no. 2, pp. 132-137, 1998.

[3] K. Demeter and Z. Matyusz, "The impact of lean practices on inventory turnover," International Journal of Production Economics, 2010.

[4] V. Crute, Y. Ward, S. Brown, and A. Graves, "Implementing lean in aerospace-challenging the assumptions and understanding the challenges," Technovation, vol. 23, pp. 917-928, 2003.

[5] P. Achanga, E. Shehab, R. Roy, and G. Nelder, "Critical success factors for lean implementation within SMEs," Journal of Manufacturing Technology Management, vol. 17, no. 4, pp. 460-471, 2006.

[6] J. Womack and D. Jones, "System lean : Penser l'entreprise au plus juste," Village Mondial, 2ème edition, Paris, 2005.

[7] Y. Yusuf, M. Sarhadi, and A. Gunaskerana, "Agile manufacturing: The drivers, concepts and attributes," International Journal of Production Economics, vol. 62, no. 1-2, pp. 23-32, 1999.

[8] Y. Wang and K. Cullinane, "Measuring container port accessibility: An application of the principal eigenvector method (PEM)," Maritime Economics \& Logistics, vol. 10, pp. 75-89.

[9] W. Dangelmaier, M. Fischer, J. Gausemeier, M. Grafe, C. Matysczok, and B. Mueck, "Virtual and augmented reality support for discrete manufacturing system simulation," Computers in Industry, vol. 56, no 4, pp. 371-383, 2005.

[10] P. Fillatreau, J-Y. Fourquet, R-L. Bolloc'h, S. Cailhol, A. Datas, and B. Puel, "Using virtual reality and 3D industrial numerical models for immersive interactive checklists," Computers in Industry, vol. 64, pp. 1253-1262, 2013.

[11] A. Caggiano and R. Teti, "Digital manufacturing cell design for performance increase," Procedia CIRP, vol. 2, pp. 64-69, 2012.

[12] D. A. Takus and D. M. Profozich, "ARENA software tutorial," in Proc. Winter Simulation Conference, 1997, pp. 541-544.

[13] L. S. Dias, G. Pereira, P. Vik, and J. A. Oliveira, "Discrete simulation tools ranking: A commercial software packages comparison based on popularity," in Proc. 9th Annual Industrial Simulation Conference, Venice, 2011.

[14] P. L. Markt and M. H. Mayer, "WITNESS simulation software: A flexible suite of simulation tools," in Proc the Simulation Conference, 1997, vol. 7, no. 10, pp. 711-717

[15] Z. M. Bzymek, M. Nunez, M. Li, and S. Powers, "Simulation of a machining sequence using delmia/quest software," Computer-Aided Design and Applications, vol. 5, no. 1-4, pp. 401-411, 2008.

[16] A. Gurumurthy and R. Kodali, "Design of lean manufacturing systems using value stream mapping with simulation," Journal of Manufacturing Technology Management, vol. 22, no. 4, pp. 444-473, 2011.

[17] T. McDonald, V. Aken, and A. F. Rentes, "Utilising simulation to enhance value stream mapping: A manufacturing case application," International Journal of Logistics: Research and Applications, vol. 5 , no. 2, pp. 213-232, 2002

[18] C. C. Huang and S. H. Liu, "A novel approach to lean control for Taiwan-funded enterprises in mainland China," International Journal of Production Research, vol. 43, no. 12, pp. 2553-2575, 2005.

[19] F. A. Abdulmalek and J. Rajgopal, "Analyzing the benefits of lean manufacturing and value stream mapping via simulation: A process sector case study," International Journal of Production Economics, vol. 107, no. 1, pp. 223-236, 2007.

[20] B. Arnaldi, P. Fuchs, and J. Tisseau, "Chapitre 1 du volume 1 du traité de la réalité virtuelle," Les Presses de l'Ecole des Mines de Paris, 2003.

[21] T. Tolio, M. Sacco, W. Terkaj, and M. Urgo, "Virtual factory: An integrated framework for manufacturing systems design and analysis," Procedia CIRP, vol. 7, pp. 25-30, 2013.

[22] J. Goulding, W. Nadim, P. Petridis, and M. Alshawi, "Construction industry offsite production: A virtual reality interactive training environment prototype," Advanced Engineering Informatics, vol. 26, pp. 103-116, 2012

[23] J. Lee, S. Han, and J. Yang, "Construction of a computer-simulated mixed reality environment for virtual factory layout planning," Computers in Industry, vol. 62, no. 1, pp. 86-98, 2011.

[24] M. C. Leu, H. A. ElMaraghy, A. Y. Nee, S. K. Ong, M. Lanzetta, M. Putz, W. Zhu, and A. Bernard, "Model based virtual assembly simulation, planning and training," Manufacturing Technology, 2013.
[25] T. Mujber, T. Szecsi, and M. Hashmi, "Virtual reality applications in manufacturing process simulation," Journal of Materials Processing Technology, pp. 1834-1838, 2004.

[26] J. Gausemier and J. M. Frund, "AR-planning tool design flexible manufacturing systems with augmented reality," in Proc. 8th Euro Graphics Workshop on Virtual Environment, 2002, pp. 19-25.

[27] F. Doil and W. Schreiber, "Augmented reality for manufacturing planning," in Proc. the Workshop on Virtual Environment, 2003, pp. 71-76.

[28] F. Kuhl, J. Dahmann, and R. Weatherly, "Creating computer simulation systems: an introduction to the high level architecture," Prentice Hall PTR Upper Saddle River, 2000.

[29] M. Latoschik and C. Fröhlich, "Towards intelligent VR multi-layered semantic reflection for intelligent virtual environments," Proceedings of the Graphics and Applications, 2007.

[30] O. D. Troyer, F. Kleinermann, B. Pellens, and W. Bille, "Conceptual modeling for virtual reality," in Proc. 26th International Conference on Conceptual Modeling, Tutorials, Posters, Panels and Industrial Contributions, 2007, vol. 83, pp. 3-18.

[31] P. Chevaillier, T. H. Trinh, M. Barange, P. D. Loor, F. Devillers, J Soler, and R. Querrec, "Semantic modeling of virtual environments using MASCARET," in Proc. the Fourth Workshop on Software Engineering and Architectures for Realtime Interactive Systems (SEARIS), 2011, pp. 1-8.

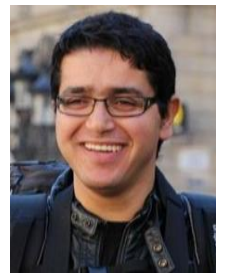

Mohamed-Amine Abidi received his engineering degree in computer science in September, 2012 from the University of Manouba, Tunisia. He is currently pursuing the $\mathrm{Ph} . \mathrm{D}$ degree in virtual reality engineering (virtual reality and simulation) at the National Engineering School of Saint-Etienne (ENISE), France. From 2012, his has been working as a lecturer at ENISE. He is the author of two papers and the co-author of one book.

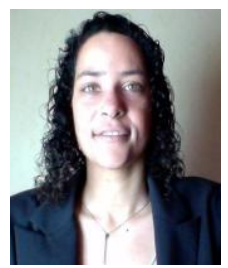

Barbara Lyonnet is an associate professor at the University of Nantes in LEMNA Laboratory, France. She has a Ph.D thesis on industrial systems. Her research interests focus on lean manufacturing, production processes and logistics. She has published in the field of engineering applications, risks, critical resources and lean manufacturing. She is the author or co-author of many research articles and some books.

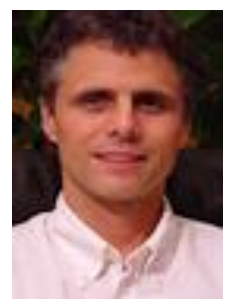

Pierre Chevaillier is a researcher in computer science at the Lab-STICC, UMR CNRS 6285. He is also a professor in computer science at the Department of Software Engineering at ENIB (Ecole Nationale d'ingénieurs de Brest). Since 2008 to 2011, he was the director of the CERV (European Center for Virtual Reality). Since 2014, he is the director of research at ENIB. He is the author or co-author of many research articles and one book.

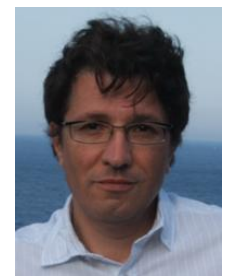

Rosario Toscano received his engineering degree from the Conservatoire National des Arts et Metiers (CNAM) in March 1994. He received his master degree with specialization in control in June 1996. He received the $\mathrm{Ph} . \mathrm{D}$ degree from the Ecole Centrale de Lyon in November 2000. He received the HDR degree from University of Jean Monnet of Saint-Etienne in December 2007. He is currently a professor at the Ecole Nationale d'Ingenieurs de Saint-Etienne (ENISE). He is the author or co-author of about sixty research articles and five books. 